\title{
British Society for Rheumatology Biologics Register
}

\author{
A Silman, D Symmons, D G I Scott, I Griffiths
}

Ann Rheum Dis 2003;62(Suppl II):ii28-ii29

The British Society for Rheumatology (BSR) established a register of patients newly treated with biological agents, the BSR Biologics Register (BSRBR), which became active in January 2002. The goal is to register all patients in the United Kingdom with rheumatic diseases, newly starting treatment with these agents and to follow them up to determine the incidence of any short and long term hazards to health. The Register is also recruiting a comparison cohort of patients with rheumatoid arthritis treated with standard disease modifying antirheumatic drugs to determine the relative contributions of disease factors and other treatments apart from biological agents on any risks observed.

$\mathrm{D}$ ata on the risk of adverse events after treatment with biological agents in patients with rheumatoid arthritis (RA) have either come from follow up of subjects recruited in clinical trials or from pharmacovigilance systems. Small numbers and selection factors in trial recruitment limit clinical trial data, even with extended follow up. Spontaneous notification by prescribers of adverse events using a national pharmacovigilance system lacks a comparison group and may be associated with significant underreporting, particularly if the event occurred a considerable time after treatment ended. Furthermore, increasingly, patients will be treated with multiple agents, and single drug registries, maintained by the relevant pharmaceutical company, will not be appropriate to answer the question of the risk associated with exposure to more than one biological agent. More importantly, many of the adverse events, which give rise to the greatest concernfor example, lymphoma, occur at increased incidence in patients with RA, especially those with severe and longstanding disease and, possibly, in those with prior exposure to immunosuppressant disease modifying antirheumatic drugs (DMARDs). For all these reasons it is essential to gather data on large cohorts of unselected patients with RA, both those newly treated with a biological agent and those with equivalent disease duration and severity treated with conventional agents. The British Society for Rheumatology Biologics Register (BSRBR) was therefore established to collect data from appropriate cohorts of patients to answer these questions.

\section{REGISTRATION OF SUBJECTS TREATED WITH BIOLOGICAL AGENTS}

The BSR issued guidelines to all rheumatologists on the use of biological agents in the treatment of RA and stated that enrolment on a national register should be considered an essential part of the prescribing process. The BSR later established such a register. The report of the UK's National Institute for Clinical Excellence (NICE) in April $2002^{1}$ granted approval for the use of tumour necrosis factor blockers for the treatment of severe RA in the National Health Service and also explicitly stated that prescription of these drugs should be accompanied by registration with the BSRBR. These points have been supported by the manufacturers of the drugs who have delegated to the BSR the task of long term surveillance for adverse events to generate the data required by regulatory authorities. All UK rheumatologists now ask such patients to consent to have their details entered on the Register and to provide both baseline and follow up data. Details of all the registration forms are available on the BSRBR website which can be visited at http://www.arc.man.ac.uk/webbiologicsreg.htm. Rheumatologists complete these forms and send them back to the Register. Currently we are developing an electronic system for data transfer.

\section{REGISTRATION OF SUBJECTS TREATED WITH NON-BIOLOGICAL AGENTS}

We have recruited seven centres throughout the UK that have well established clinical databases of subjects with RA. In each of these centres all patients starting or changing to a new DMARD are considered for eligibility for recruitment to the comparison cohort. The aim is to derive cohorts with sufficient overlap in their disease severity, as assessed by the 28 joint disease activity score (DAS 28), Health Assessment Questionnaire (HAQ), and disease duration, to make it possible to adjust for any differences when determining the relative risks of different adverse outcomes between the groups.

\section{FOLLOW UP}

Follow up is multifaceted. Firstly, the rheumatologist notifying each subject is contacted every six months and asked about the development of any serious adverse events, including any admissions to hospital and serious infection. The subjects themselves are given a diary every six months to record all serious adverse events, similarly defined. All subjects are also "flagged" at the Office for National Statistics to ascertain mortality and any new cases of cancer. The recording of any serious event will be followed by chart review to establish the exact diagnosis, duration, and consequences.

\section{OWNERSHIP OF THE BSRBR}

The BSR owns the Register and controls access to the data and its release. It has set up a management committee and data monitoring board to advise the ARC Epidemiology Unit, the group charged with carrying out the Register functions, on all aspects of data collection and analysis. The Register is funded by grants from the relevant pharmaceutical companies to the BSR, but the companies have rights of access to the data on their own product(s), particularly to those data items the companies are required to submit to regulatory authorities.

\section{SIZE OF THE REGISTER}

The Register became operational in January 2001, but the number of subjects entered increased substantially after the NICE report in April 2002. As of April 2003 over 2500 patients

Abbreviations: BSRBR, British Society for Rheumatology Biologics Register; DMARDs, disease modifying antirheumatic drugs; NICE, National Institute for Clinical Excellence; RA, rheumatoid arthritis 
were enrolled on the register. The number of new registrations of subjects receiving biological agents is approaching 300 a month and is still increasing. The control centres have only become operational during the past six months and it is hoped that, from the seven centres, it will be possible to recruit similar numbers of subjects treated with non-biological agents. With these numbers BSRBR should have the power within a short period of time to examine the key questions it was charged with answering. The data will also permit an evaluation of the relative costs and benefits of the use of these drugs in everyday rheumatological practice.

\section{Authors' affiliations}

A Silman, D Symmons, ARC Epidemiology Unit, Manchester, UK

D G I Scott, I Griffiths, British Society for Rheumatology, UK

Correspondence to: Professor A J Silman, ARC Epidemiology Unit, Stopford Building, University of Manchester, Oxford Road, Manchester M13 9PT, UK; alan.silman@man.ac.uk

\section{REFERENCE}

1 National Institute for Clinical Excellence. Guidance on the use of etanercept and infliximab for the treatment of rheumatoid arthritis. NICE Technology Appraisal. London; NICE 2002:36.

\section{Get published within days of acceptance with ARD}

We are delighted to announce that the Annals of the Rheumatic Diseases is launching a "publish ahead of print" programme from February 2004. Selected papers will be fast tracked and published online months before they appear in the print journal.

Papers of major significance to the international rheumatology community will be published within days of acceptance. The published article will be the raw accepted manuscript; edited and typeset versions will also be published as soon as they are available.

In addition to being available on ARD Online, the publish ahead of print articles will be searchable through PubMed/Medline-establishing primacy for your work. They will be linked from the ARD Online home page.

$A R D$ 's publish ahead of print programme is unique among the major rheumatology journals - to take advantage of this service submit your papers to Annals of the Rheumatic Diseases using our online submission and review system Bench>Press (http://submitard.bmijournals.com). For further information contact ARD@bmigroup.com. 